\title{
Depressão e comorbilidade: um caso clínico*
}

Teresa Ventura**

\section{RESUMO}

Introdução: É frequente a coexistência de depressão com doenças crónicas. Esta associação leva a pior evolução, tanto do quadro psiquiátrico, como da doença crónica. Contudo, nem sempre o diagnóstico da depressão é evidente e, mesmo quando correctamente identificada, muitas vezes é subtratada, quer por razões imputáveis ao médico, quer por má adesão do doente à terapêutica.

Descrição do caso: Maria é grande utilizadora da consulta, a que recorre por tonturas, acufenos, fadiga e dores osteoarticulares. Os problemas de saúde identificados incluem entidades com potencial papel etiológico nestes sintomas, que a doente hipervaloriza. No entanto, esta cumpria irregularmente a terapêutica farmacológica e mantinha hábitos de vida prejudiciais ao controlo desses problemas de saúde.

Após múltiplas consultas de padrão semelhante, admitiu, quando questionada, irritabilidade, anedonia, tendência para o isolamento social, hipersónia e humor depressivo que atribuía ao sofrimento físico.

Após várias tentativas de introdução de terapêutica antidepressiva, que a doente abandonava após escassos dias alegando efeitos secundários, a marcação de consultas quinzenais resultou em maior adesão à terapêutica, antidepressiva e outra, menos queixas e melhor controlo das suas doenças crónicas.

Comentário: A ênfase nos sintomas somáticos e o facto de serem explicáveis no contexto da comorbilidade da doente e, assim, retirados como critérios de suspeição de depressão, levaram a que esta se mantivesse mascarada ao longo do tempo.

O médico deve ter presente a frequência da depressão associada a doenças crónicas, de modo a conseguir melhor acuidade diagnóstica. A abordagem inclusiva, que contabiliza sintomas independentemente de serem explicáveis pela patologia coexistente, diminui o risco de não se diagnosticar um quadro depressivo oligossintomático, não obstante poder gerar falsos-positivos. Pelo contrário, a abordagem excludente utilizada durante algum tempo, adiou o diagnóstico (falso-negativo) e o tratamento, com repercussões no controlo da globalidade dos problemas de saúde e na qualidade de vida da doente.

Palavras-chave: Depressão; Doença Crónica.

\section{INTRODUÇÃO}

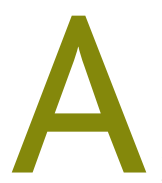

depressão é apontada como a perturbação que causa a maior proporção de incapacidade a nível mundial, liderando a lista das patologias responsáveis pelo peso global da doença. ${ }^{1}$

Doenças físicas podem contribuir para a patogénese da depressão, seja através de efeitos directos, da doença ou dos fármacos prescritos para a tratar, na função cerebral, seja através das suas repercussões psicossociais. $^{2}$

*Os nomes referidos no relato de caso são fictícios.

**Chefe de Serviço/Assistente Graduada Sénior do Centro de Saúde Santo Condestável. Assistente Convidada do Departamento de Medicina Geral e Familiar da Faculdade de Ciências Médicas da Universidade Nova de Lisboa.
A associação entre doença crónica e depressão é bidireccional: não só as doenças físicas (ou o seu tratamento) podem desencadear ou exacerbar perturbações depressivas, mas também estas podem precipitar ou agravar doenças fisicas crónicas. ${ }^{3,4}$ No entanto, muitas vezes, a depressão e a doença física coexistem sem que se possa definir claramente a existência e o sentido duma eventual doença causal.

A prevalência da depressão tem sido fortemente associada com o número de doenças crónicas - quanto maior for a comorbilidade, maior a frequência da sintomatologia depressiva. ${ }^{4-6}$

O diagnóstico de depressão nos pacientes é frequentemente difícil porque vários sinais e sintomas relevantes podem ser confundidos com aqueles que decorrem do facto de se ter uma doença física. ${ }^{?}$ 
O caso clínico que se apresenta neste artigo foi seleccionado por se ter considerado:

- Demonstrativo da associação conhecida entre depressão e doença crónica, ainda mais frequente em situações de comorbilidade;

- Exemplificador da circularidade de efeitos, que implica pior evolução, tanto da depressão, como das doenças crónicas coexistentes;

- Evidenciador das dificuldades que por vezes ocorrem no diagnóstico atempado da depressão.

A apresentação do caso foi estruturada de modo a servir os seguintes objectivos:

- Demonstrar as dificuldades do diagnóstico diferencial nas situações em que os sintomas de depressão são comuns a doenças físicas;

- Mostrar como a depressão pode induzir menor adesão às orientações terapêuticas, comprometendo o controlo das doenças crónicas;

- Delinear estratégias que aumentem a adesão às orientações terapêuticas.

\section{DESCRIÇÃO DO CASO}

1. Dados demográficos, antecedentes e contexto

\section{familiares}

Maria tem 69 anos, é viúva e vive sozinha. Antiga telefonista, reformou-se há quatro anos. Pertence à classe IV de Graffar.

Do seu genograma (figura 1) ressaltam os seguintes dados:

- Uma história familiar positiva para neoplasia do cólon (dois irmãos) e da mama (irmã falecida em 2007), para diabetes mellitus (irmã) e para doença cardiovascular (a mãe e três irmãos), o que deve implicar atenção acrescida nos procedimentos preventivos a incluir no plano terapêutico de Maria.

- O pai com problemas de alcoolismo, protagonizando cenas de violência doméstica, a evolução pessoal alcoólica de pelo menos três irmãos e o casamento com parceiro alcoólico da Maria, no ano em que o pai faleceu.

- A Maria não vive apenas sozinha, vive muito só, sendo de realçar:

- a relação conflituosa ou distante com os únicos irmãos vivos - no seu dizer, a Teresa desde pequena tem a mania que manda nela e o Francisco é alcoólico e irresponsável como o pai. A irmã com que tinha uma relação melhor morreu há três anos com cancro da mama metastizado;

- também com a filha, que viveu com ela até aos 35 anos e presentemente reside no Alentejo, a relação é conflituosa, desde que Maria se opôs ao seu casamento com o actual marido;

- das netas fala com afecto, mas quando impelida a tentar uma aproximação maior com estas, responde que o Alentejo a irrita e elas raramente se deslocam a Lisboa;

- com o genro a relação sempre foi distante;

- costumava acompanhar a cunhada em alguns programas de saídas, mas ultimamente não tolera algumas características da personalidade desta, pelo que se tem afastado.

- O marido faleceu jovem, quatro anos após o casamento com a Maria.

\section{Dados da história clínica}

Maria é uma grande utilizadora de consultas e os motivos para tal são repetitivos. Queixa-se de:

- Tonturas, nomeadamente quando está sentada ou deitada e, de forma rápida, assume a posição ortostática; também refere episódios de desequilíbrio, que descreve como «sentir o chão fugir-lhe dos pés»

- Acufenos

- Fadiga

- Dores osteoarticulares, sobretudo lombalgias.

Visando uma melhor caracterização do caso, seleccionaram-se, para apresentação, dados registados na nota de seguimento de uma destas consultas, quase idênticas entre si.

Os dados subjectivos, registados em Setembro de 2009, incluem, para além das queixas atrás referidas, menção a uma alimentação desequilibrada, com apenas três refeições diárias, à base de comida de snack e muitos doces por, numa citação da doente, «falta de paciência para preparar refeições».

Existem ainda referências a sedentarismo, ao consumo de cerca de 30 cigarros diários (mais do que o registado três anos antes) e à toma irregular dos medicamentos prescritos «por esquecimento» (sic).

Não tinha realizado os exames complementares de diagnóstico requisitados na consulta anterior.

Sendo diabética, tinha ido a consulta de oftalmologia para exclusão de retinopatia, tendo o exame sido normal. 


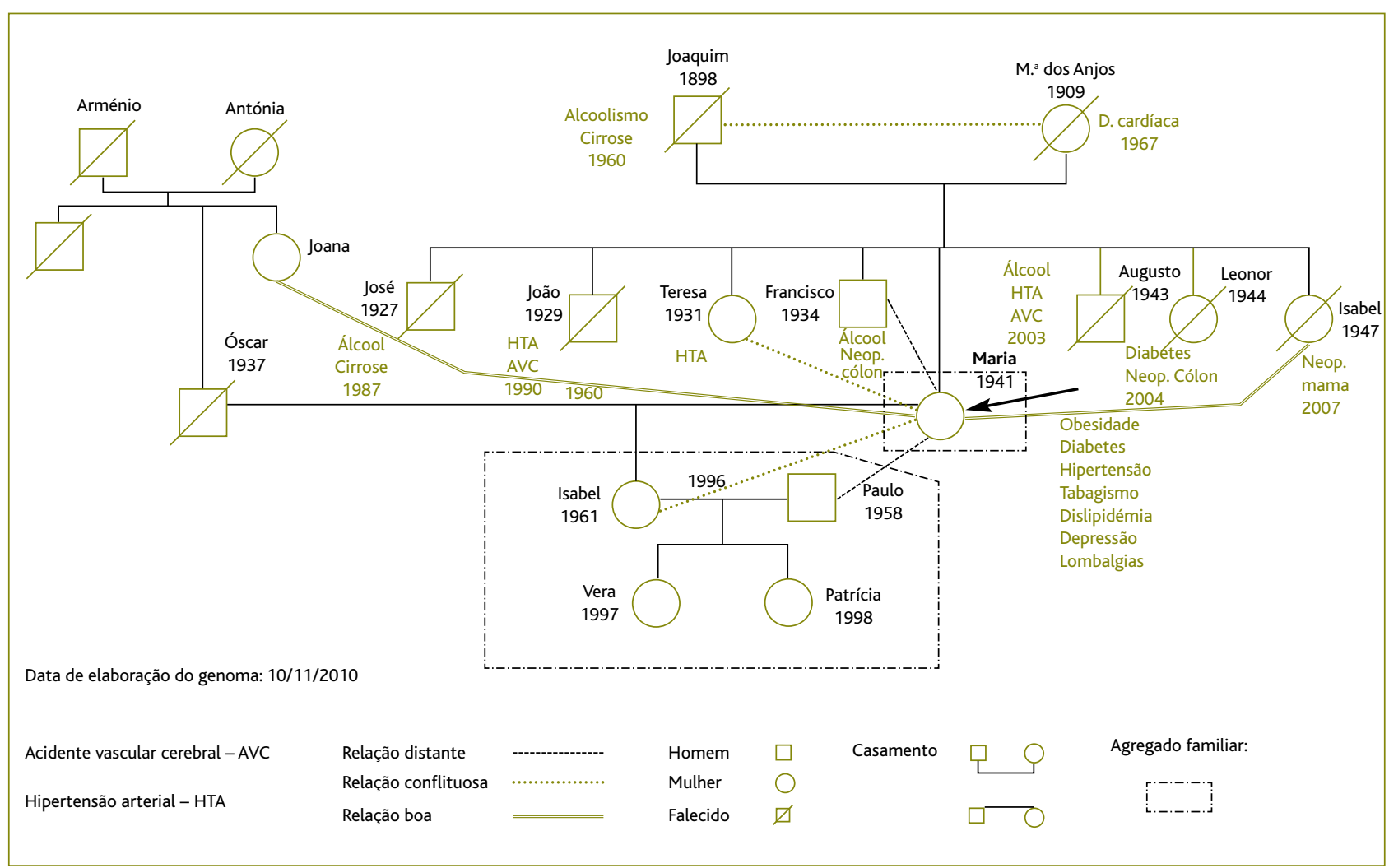

Figura 1. Genograma.

Foi ainda registado um grande consumo de ácido acetilsalicílico (cerca de 3 gramas diários) como analgésico para alívio das dores osteoarticulares.

Os dados objectivos desta mesma consulta incluíam valores tensionais de 162/105 mm Hg, um índice de massa corporal (IMC) de 31 com perímetro abdominal elevado, denotando obesidade central.

$\mathrm{O}$ restante exame objectivo dirigido às queixas era normal.

A glicémia capilar determinada pela enfermeira, antes da consulta, era de $223 \mathrm{mg} / \mathrm{dL}$.

Os problemas de saúde activos registados eram:

- Obesidade

- Diabetes tipo 2

- Tabagismo

- Hipertensão complicada, nomeadamente por hipertrofia ventricular esquerda

- Microalbuminúria elevada

- Dislipidémia

- Espondilartrose da coluna lombar e cervical

\section{Raciocínio clínico}

O raciocínio adoptado era o da explicação das queixas da Maria no contexto da lista de problemas já identificados:

As tonturas quando se põe de pé de forma rápida foram interpretadas como hipotensão ortostática, comum nos idosos, nos hipertensos e nos doentes a fazer inibidores da enzima de conversão da angiotensina, antagonistas do cálcio ou diuréticos, para nomear apenas as situações que se verificam nesta doente.

As variações dos valores tensionais e do índice glicémico, as alterações das três primeiras vértebras cervicais, bem como o possível salicilismo foram considerados etiologias possíveis das vertigens.

A hipertensão, a diabetes, a cervicartrose, efeitos adversos dos antihipertensores que a doente fazia e o hipotético salicilismo poderiam explicar os acufenos.

A diabetes, a hipertensão, o tabagismo e os erros dietéticos foram considerados explicações possíveis para a fadiga. 
A espondilartrose e vícios posturais estariam na base das lombalgias.

\section{Estratégias terapêuticas}

A estratégia seguida em múltiplas consultas, foi a de fazer a doente associar os sintomas que hipervalorizava aos problemas de saúde identificados, de modo a conseguir um contrato terapêutico, nomeadamente no que se relaciona com as alterações de hábitos de vida e cumprimento da terapêutica farmacológica.

Verificando-se um consumo excessivo de salicilatos, os riscos de efeitos adversos gastrointestinais e a hipótese de salicilismo como etiologia das vertigens e dos acufenos, levaram a que se mantivesse uma dose de 100 mg para anti-agregação plaquetária, sendo prescrito paracetamol como analgésico. Reforçou-se a educação postural, tendo a doente recusado tratamentos de fisioterapia, referindo não ter paciência.

Estas estratégias revelaram-se ineficazes, sucedendo-se consultas de padrão idêntico ao longo do tempo.

\section{Diagnóstico e tratamento de depressão}

Foram a resistência à terapêutica e a desproporção entre a intensidade dos sintomas e o esperado pelo quadro clínico que levaram à suspeição de depressão.

Assim, foi mudado o guião da entrevista clínica habitual com a Maria que admitiu, quando especificamente questionada, irritabilidade, anedonia, tendência para o isolamento social, hipersónia, ingestão compulsiva de alimentos açucarados e humor depressivo que atribuía ao sofrimento físico. Reconheceu não gostar de viver sozinha e não encontrar objectivos de vida.

Consequentemente, foi instituída terapêutica antidepressiva. Na selecção do antidepressivo, a opção recaíu no cloridrato de bupropion, na dose de $150 \mathrm{mg}$ diários, tendo em conta a cessação tabágica pretendida e os efeitos facilitadores do fármaco na pressecução desse objectivo. Esta opção foi assumida, não obstante ter presente a doente estar a fazer antidiabéticos orais, que reduzem o limiar de convulsão, um dos efeitos adversos do cloridrato de bupropion.

A prescrição de antidepressivo foi acompanhada por uma atitude de ajuda na identificação e verbalização dos sentimentos, fornecendo-lhes suporte, e de estímulo à criação duma rede familiar e social capaz de lhe proporcionar uma protecção eficaz.
Na consulta seguinte a estas decisões, ocorrida dois meses depois, a doente, apenas quando interrogada, referiu o abandono do cloridrato de bupropion dois dias após o seu início, por nervosismo, agitação e secura de boca.

Mantinha as queixas habituais e o cumprimento do plano terapêutico dirigido aos seus múltiplos problemas de saúde era apenas parcial e irregular.

O bupropion foi substituído por $50 \mathrm{mg}$ diários de sertralina.

Maria também abandonou a sertralina, alegando náuseas. Foi então negociado com ela a reintrodução do fármaco com fraccionamento de comprimidos e aumento progressivo da dose. Em caso de algum efeito secundário que, embora previsivelmente transitório, ela considerasse não conseguir tolerar, comprometeu-se a não interromper a terapêutica e a contactar a médica para, juntas, decidirem a atitude a tomar.

Durante três meses foram programadas consultas quinzenais, visando o reforço da adesão à terapêutica, antidepressiva e outra. Para cada quinzena foram negociadas pequenas mudanças concretas e as metas foram sendo revistas sempre que uma mudança parcial ocorria.

\section{Situação actual}

Seis meses após a introdução da sertralina no plano terapêutico, Maria apresenta menos queixas, maior adesão à terapêutica e algumas mudanças benéficas nos hábitos de vida.

Retomou, ainda, o contacto com a cunhada, embora continue a resistir a diversificar actividades e a alargar a rede social e familiar.

\section{COMENTÁRIO}

No caso da Maria, apenas após a falência do modelo biomédico tradicional e surgimento da suspeita de depressão, foi alargado o campo de observação, de modo a aprofundar a história psicossocial da paciente, tendo a elaboração do genograma sido um instrumento importante. Ficou a percepção de que:

- A vivência familiar na infância, como é típico das famílias alcoólicas, determinou:

- Fraca resiliência;

- Dificuldades em identificar e exprimir emoções, com exteriorização, quando muito, dos sentimen- 
tos acusatórios, o que faz com que as dificuldades emocionais se exprimam por equivalentes somáticos;

- Pouca capacidade em envolver-se em relações próximas e estáveis, o que se reflectiu na construção duma escassa rede de suporte social e familiar ao longo da vida;

- Rigidez no funcionamento, com as dificuldades inerentes de adaptação às mudanças exigidas pelos acontecimentos vitais;

- Vulnerabilidade à depressão.

- O efeito cumulativo de crises não resolvidas, normativas (como a saída da filha de casa, quando casou aos 35 anos, ou a reforma) e acidentais (como a morte do marido ainda jovem e da maioria dos elementos da sua família de origem ou, ainda, o aparecimento das suas próprias doenças) levaram à evolução depressiva.

Vários estudos têm identificado um conjunto de factores de risco sociais, físicos e demográficos para a depressão em fases mais tardias da vida. ${ }^{8}$ Muitos destes factores estão presentes no caso da Maria, nomeadamente:

- Género feminino; ${ }^{9}$

- Idade, ${ }^{10,11}$ embora alguns estudos tenham verificado que o seu efeito sobre a depressão desaparece após outras variáveis sóciodemográficas e de saúde terem sido controladas; ${ }^{10,12,13}$

- Viuvez; ${ }^{8,9,14}$

- Reforma; ${ }^{9}$

- Viver só; ${ }^{9}$

- Grau de escolaridade; ;,14-16

- Baixo nível sócio-económico; ${ }^{8,9,17,18}$

- Estrutura de personalidade neurótica; ${ }^{9}$

- Ocorrência de acontecimentos de vida desgastantes; ${ }^{9,16}$

- Falta de confidente/relação íntima ${ }^{9,11,19,20}$ e de suporte social; $9,11,16,21$

- Disfunção familiar, com baixo apoio emocional por parte da filha; ${ }^{9,11}$

- Escassez de actividade sociais; ${ }^{9,22}$

- Existência de doenças físicas, ${ }^{8,9,11,23,24}$ sua cronicida$\mathrm{de}^{3,4,25,26}$ e número de doenças. ${ }^{4-6,19}$ Determinadas doenças crónicas, incluindo a diabetes ${ }^{9,23,25}$ e a hipertensão, ${ }^{9,25}$ presentes no caso da Maria, têm sido associadas a depressão.
No caso em estudo, a não valorização de sintomas de depressão comuns aos problemas coexistentes retardaram o diagnóstico.

A questão da validade dos sintomas somáticos como critérios de depressão foi objecto dum estudo longitudinal que acompanhou, durante seis meses, quase 500 deprimidos, com e sem comorbilidade física. Os autores concluem que os sintomas somáticos devem ser válidos para o diagnóstico de depressão e que melhoram da mesma maneira nos doentes com e sem doenças físicas crónicas. ${ }^{27}$

Já Cavanaugh ${ }^{28}$ propõe uma abordagem na qual sintomas como fadiga e alterações do sono, apetite, peso e alterações psicomotoras ajudam a corroborar o diagnóstico de depressão quando excessivas relativamente ao esperado pela condição física e seus tratamentos e quando surgem associadas temporalmente a sintomas cognitivo-afectivos (humor depressivo, anedonia).

No caso da Maria, o impacto da depressão no controlo das doenças coexistentes está de acordo com o que a investigação tem demonstrado de forma consistente: indivíduos com doenças físicas que estão deprimidos apresentam menor adesão aos tratamentos propostos, ${ }^{29}$ maior insuficiência de autocuidados, ${ }^{30,31}$ maior incapacidade funcional, ${ }^{32}$ menor qualidade de vida, ${ }^{33}$ aumento de custos com a saúde ${ }^{34}$ e pior prognóstico, com maiores morbilidade ${ }^{35}$ e mortalidade..$^{35,37}$

Dada a prevalência da depressão em doentes crónicos e as suas implicações, o médico deve ser pró-activo na identificação de sintomatologia depressiva. Apesar de existirem escalas para a sua detecção, algumas validadas em amostras de determinados grupos da população portuguesa, estas são mais utilizadas no ensino e na investigação. Com menor utilização na prática clínica, existe mesmo quem as desaconselhe neste contexto, como Letícia Furlanetto, ${ }^{38}$ que refere o risco de se começar a medicar o papel em vez do sujeito.

Do caso apresentado, conclui-se:

- O médico deve ter presente a frequência da depressão associada a doenças crónicas, de modo a conseguir melhor acuidade diagnóstica.

- A abordagem inclusiva, que contabiliza sintomas independentemente de serem explicáveis pela patologia coexistente, diminui o risco de não se diagnosticar um quadro depressivo oligossintomático, não obstante poder gerar falso-positivos. Pelo contrário, 
a abordagem excludente utilizada durante algum tempo, adiou o diagnóstico (um falso-negativo) e o tratamento.

- No caso de morbilidade mascarada, as queixas depressivas podem manifestar-se apenas quando especificamente interrogadas.

- A melhoria da depressão é fundamental no controlo da globalidade dos problemas de saúde e na qualidade de vida do doente.

- Uma intervenção inicial intensiva, com mais de uma consulta por mês, poderá ser necessária para obter adesão terapêutica.

\section{REFERÊNCIAS BIBLIOGRÁFICAS}

1. Murray CJL, Lopez AD. The global burden of disease: a comprehensive assessment of mortality and disability from diseases, injuries, and risk factors in 1990 and projected to 2020. Cambridge (MA): Harvard University Press; 1996.

2. Alexopoulos GS, Buckwalter K, Olin J, Martinez R, Wainscott C, Krishnan KR. Comorbidity of late life depression: an opportunity for research on mechanisms and treatment. Biol Psychiatry 2002 Sep 15; 52 (6): 543-58.

3. Polsky D, Doshi JA, Marcus S, Oslin D, Rothbard A, Thomas N, et al. Longterm risk for depressive symptoms after a medical diagnosis. Arch Intern Med 2005 Jun 13; 165 (11): 1260-6.

4. Duarte MB, Rego MA. Comorbilidade entre depressão e doenças clínicas em um ambulatório de geriartria. Cad Saúde Pública 2007 Mar; 23(3): 691-700.

5. Mills TL. Comorbid depressive symptomatology: isolating the effects of chronic medical conditions on self-reported depressive symptoms among community-dwelling older adults. Soc Sci Med 2001 Sep; 53 (5): 569-78.

6. Bisschop MI, Kriegsman DM, Deeg DJ, Beekman AT, van Tilburg W. The longitudinal relation between chronic diseases and depression in older persons in the community: the Longitudinal Aging Study Amsterdam. J Clin Epidemiol 2004 Feb; 57 (2): 187-94.

7. Furlanetto LM. Diagnóstico. In: Fráguas Júnior R, Figueiredo JAP, editores. Depressão em medicina interna e em outras condições médicas. Depressões secundárias. Rio de Janeiro: Atheneus; 2000. p.11-20.

8. Osborn D, Fletcher A, Smeeth L, Stirling S, Bulpitt CJ, Breeze E, et al. Factors associated with depression in a representative sample of 14 217 people aged 75 and over in United Kingdom: results from the MRC trial of assessment and management of older people in the community. Int J Geriatr Psychiatry 2003 Jul; 18 (7): 623-30.

9. Vaz SF. A depressão no idoso institucionalizado: estudo em idosos residentes nos lares do distrito de Bragança. [Dissertação de Mestrado apresentada à Faculdade de Psicologia e Ciências de Educação da Universidade do Porto]. Porto: Faculdade de Psicologia e Ciências de Educação da Universidade do Porto; 2009. Disponível em: http://repositorio-aberto.up.pt/bitstream/10216/23338/2/A\%20depressao\%20no\% 20idoso\%20institucionalizado.pdf [acedido em 31/12/2010].

10. Zarit SH, Femia EE, Gatz M, Johansson B. Prevalence, incidence and correlates of depression in the oldest old: the OCTO study. Aging Mental Health 1999; 3 (2): 119-28.

11. Zunzunegui M, Béland F, Llácer A, León V. Gender differences in depressive symptoms among Spanish elderly. Social Psychiatry Psychia- tr Epidemiol 1998 May; 33 (5): 195-205.

12. Blazer DG 2nd, Hybels CF. Origins of depression in later life. Psychol Med 2005 Sep; 35 (9): 1241-52.

13. Jones RN, Marcantonio ER, Rabinowitz T. Prevalence and correlates of recognized depression in U.S. nursing homes. J Am Geriatr Soc 2003 Oct; 51 (10): 1404-9.

14. Papadopoulos FC, Petridou E, Argyropoulou S, Kontaxakis V, Dessypris $\mathrm{N}$, Anastasiou A, et al. Prevalence and correlates of depression in late life: a population based study from a rural Greek town. Int J Geriatr Psych 2005 Apr; 20 (4): 350-7.

15. Ganguli M, Dube S, Johnston JM, Pandav R, Chandra V, Dodge HH. Depressive symptoms, cognitive impairment and functional impairment in a rural elderly population in India: a Hindi version of the geriatric depression scale (GDS-H). Int J Geriatr Psych 1999 Oct; 14 (10): 807-20.

16. Beekman AT, Deeg DJ, van Tilburg T, Smit JH, Hooijer C, van Tilburg W. Major and minor depression in later life: a study of prevalence and risk factors. J Affect Disorders 1995 Dec 24; 36 (1-2): 65-75.

17. Bazargan M, Hamm-Baugh VP. The relationship between chronic illness and depression in a community of urban black elderly persons. J Gerontol B Psychol Sci Social Sci 1995 Mar; 50 (2): S119-27.

18. Blazer D, Burchett B, Service C, George LK. The association of age and depression among the elderly: an epidemiologic exploration. J Gerontol 1991 Nov; 46 (6): M210-15.

19. Kennedy GJ, Kelman HR, Thomas C, Wisniewski W, Metz H, Bijur PE. Hierarchy of characteristics associated with depressive symptoms in an urban elderly sample. Am J Psych 1989 Feb; 146 (2): 220-5.

20. Evans S, Katona C. Epidemiology of depressive symptoms in elderly primary care attenders. Dementia 1993 Nov-Dec; 4 (6): 327-33.

21. Prince MJ, Harwood RH, Blizard RA, Thomas A, Mann AH. Impairment, disability and handicap as risk factors for depressive in old age. The Gospel Oak Project V. Psychol Med 1997 Mar; 27 (2): 311-21.

22. Bergdahl E, Allard P, Alex M, Lundman B, Gustafson Y. Gender differences in depression among the very old. Int Psychogeriatr 2007 Dec; 19 (6): 1125-40.

23. Chou KL, Chi I. Prevalence and correlates of depression in Chinese oldest-old. Int J Geriatr Psychiatry 2005 Jan; 20 (1): 41-50.

24. Moldin SO, Scheftner WA, Rice JP, Nelson E, Knesevich MA, Akiskal H. Association between major depressive disorder and physical illness. Psychol Med 1993 Aug; 23 (3): 755-61.

25. Sherina MS, Zulkefli N, Mustaqim A. Prevalence of depression with chronic illness among the elderly in a rural community in Malasya. Asia Pacific Fam Med 2003 Oct-Dec; 2 (4): 196-9.

26. Barrett JE, Barret JA, Oxman TE, Gerber PD. The prevalence of psychiatric disorders in a primary care practice. Arch Gen Psychiatry $1988 \mathrm{Dec}$; 45 (12): 1100-6.

27. Simon GE, Von Korff M. Medical co-morbidity and validity of DSM-IV depression criteria. Psychol Med 2006 Jan; 36 (1): 27-36.

28. Von Ammon Cavanaugh S. Depression in the medically ill. Critical issues in diagnostic assessment. Psychosomatics 1995 Jan-Feb; 36 (1): 48-59.

29. Lustman PJ, Clouse RE. Depression in diabetic patients: the relationship between mood and glycemic control. J Diabetes Complications 2005 Maar-Apr; 19 (2): 113-22.

30. Ziegelstein RC. Depression in patients recovering from a myocardial infarction. JAMA 2001 Oct 3; 286 (13): 1621-7.

31. Lin EH, Katon W, Von Korff M, Rutter C, Simon GE, Oliver M, et al. Relationship of depression and diabetes self-care, medication adherence, and preventive care. Diabetes Care 2004 Sep; 27 (9): 2154-60.

32. Sullivan MD, LaCroix AZ, Baum C, Grothaus LC, Katon WJ. Functional status in coronary artery disease: a one-year prospective study of the 
role of anxiety and depression. Am J Med 1997 Nov; 103 (5): 348-56.

33. De Jonge P, Kempen $\mathrm{Gl}$, Sanderman R, Ranchor AV, van Jaarsveld $\mathrm{CH}$, van Sonderen $\mathrm{E}$, et al. Depressive symptoms in elderly patients after a somatic illness event: prevalence, persistence and risk factors. Psychosomatics 2006 Jan-Feb; 47 (1): 33-42.

34. Unützer J, Patrick DL, Simon G, Grembowski D, Walker E, Rutter C, et al. Depressive symptoms and the cost of health services in $\mathrm{HMO}$ patients aged 65 years and older: a 4-year prospective study. JAMA 1997 May 28; 277 (20): 1618-23.

35. Lespérance F, Frasure-Smith $N$, Juneau M, Théroux P. Depression and 1-year prognosis in unstable angina. Arch Intern Med 2000 May 8; 160 (9): 1354-60.

36. Furlanetto LM, von Ammon Cavanaugh S, Bueno JR, Creech SD, Powell $\mathrm{LH}$. Association between depressive symptoms and mortality in medical patients. Psychosomatics 2000 Sep-Oct; 41 (5): 426-32.

37. Rumsfeld JS, Jones PG, Whooley MA, Sullivan MD, Pitt B, WeintraubWS, et al. Depression predicts mortality and hospitalization in patient with myocardial infarction complicated by heart failure. Am Heart J 2005 Nov; 150 (5): 961-7.
38. Furlanetto LM, Del Moral JA, Gonçalves AH, Rodrigues K, Jacomino ME. Diagnosticando depressão em pacientes internados com doenças hematológicas: prevalência e sintomas associados. J Bras Psiquiatr 2006; 55 (2): 96-101. Disponível em: http://www.scielo.br/pdf/jbpsiq/v55n2/ v55n2a01.pdf [acedido em 31/12/200].

\section{CONFLITOS DE INTERESSE}

Declaro não ter interesses financeiros, ligações pessoais ou outros conflitos de interesse que possam enviesar o presente trabalho.

\section{ENDEREÇO PARA CORRESPONDÊNCIA}

Teresa Ventura

Rua Joaquim Quirino, n. 6 - 11. ${ }^{\circ}$ Direito

2770-089, Paço d'Arcos, Oeiras

E-mail: theresa.v@sapo.pt

Recebido em 15/12/2010

Aceite para publicação em 03/01/2011

\section{ABSTRACT}

\section{DEPRESSION AND CO-MORBIDITY: CASE REPORT}

Introduction: The co-existence of depression and chronic disease is frequent. This association leads to a worse evolution, of both the psychiatric framework and the chronic disease. However, the depression diagnosis is not always evident and even when it is identified correctly it is often under-treated, either for reasons imputable to the doctor or due to the patient's poor adhesion to the therapeutics.

Description of the case: Maria frequently attends medical appointments complaining of dizziness, tinnitus, fatigue and bone and joint pains. The identified health problems include entities with a potential aetiological role in these symptoms, which are overvalued by the patient. However, the latter complied only irregularly with the pharmaceutical therapeutics and kept lifestyle detrimental to the control of such health problems.

After multiple appointments of similar pattern she admitted, upon questioning, irritability, anhedonia, tendency for social isolation, hypersomnia and depressive humour, which she attributed to her physical aflictions.

After several attempts to introduce anti-depressive medication, which the patient waived a few days later, alleging side effects, medical appointments at fortnight intervals resulted in a better compliance with the therapeutics, both anti-depressive and other, less complaints and better control of her chronic diseases.

Comment: The emphasis on the somatic symptoms and the fact that they could be explained within the context of the patient's co-morbidity, therefore withdrawn as criteria of depression suspicion, led to the masking of the latter over time.

The doctor must always consider the frequency of depression associated with chronic diseases, in order to achieve better diagnosis acuity. The inclusive approach, accounting for symptoms that are irrespective of being explained by the coexisting pathology, lessens the risk of not diagnosing an oligosymptomatic depressive framework, notwithstanding the fact that it may generate false-positive features. On the contrary, the excluding approach used for some time retarded the (false-negative) diagnosis and the treatment, with repercussions on the control of the whole health problems and on the patient's quality of life.

Keywords: Depression; Chronic Disease. 